\title{
Interface effects on the effective behavior of some Kelvin-Voigt viscoelastic heterogeneous bodies
}

\author{
C. Licht ${ }^{\mathrm{a}, \mathrm{b}, \mathrm{c}, *}$ and S. Orankitjaroen ${ }^{\mathrm{b}, \mathrm{c}}$ \\ ${ }^{a}$ LMGC, UMR-CNRS 5508, Université Montpellier II, Case courier 048, Place Eugène Bataillon, \\ 34095 Montpellier cedex 5, France \\ E-mail: clicht@univ-montp2.fr \\ ${ }^{\mathrm{b}}$ Department of Mathematics, Faculty of Science, Mahidol University, Bangkok 10400, Thailand \\ ${ }^{\mathrm{c}}$ Centre of Excellence in Mathematics, CHE, Bangkok 10400, Thailand \\ E-mail: somsak.ora@mahidol.ac.th
}

\begin{abstract}
The effective behavior of a solid made from a periodic distribution of inclusions in a matrix is investigated. Inclusions and matrix are linearly elastic or viscoelastic of Kelvin-Voigt type (and possibly rigid for the inclusions) while the link between them can be pure adhesion or viscous friction with bilateral contact or involve a very thin viscoelastic layer. As long as one constituent is viscoelastic, the effective behavior is no longer of Kelvin-Voigt type but with memory.
\end{abstract}

Keywords: homogenization, Kelvin-Voigt viscoelasticity, viscoelasticity with memory, interface effects, semi-groups of operators, Laplace transform, integro-differential equations

\section{Introduction}

The effective behavior of a periodically heterogeneous body made of Kelvin-Voigt type viscoelastic materials was long ago investigated by homogenization theory in [4]. It was proven that this effective behavior is no longer of Kelvin-Voigt type but rather with fading memory. Here we extend this result to the case of composites with a periodic structure made of two or three phases, with each phase being either viscoelastic of Kelvin-Voigt type or purely elastic, but at least one is viscoelastic. The three-phase case corresponds to a periodic distribution of inclusions in a matrix linked to the matrix through a thin soft layer, or the so called interphase, whereas in the two-phase case, inclusions and matrix are perfectly bonded or a condition involving bilateral contact with friction of Kelvin-Voigt type may occur. The mechanical motivation underlying this study concerns the evident technological difficulty of achieving perfect bonding of inclusions in a matrix when manufacturing composite materials [9].

More precisely, the geometry of the composite can be described as follows. Let $Y:=(0,1)^{3}$ the unit cell of $\mathbb{R}^{3}, I$ a simply connected open set, with a Lipschitz-continuous boundary $S$, whose closure $\bar{I}$ is included in $Y$, and $M:=Y \backslash \bar{I}$. If $h$ is a small real number (less than $\operatorname{dist}(S, \partial Y)$ ), let $I_{h}=\{y \in$ $I ; \operatorname{dist}(y, S)>h\}, B_{h}=\{y \in Y ; \operatorname{dist}(y, S)<h\}$ and $M_{h}=Y \backslash\left(\bar{B}_{h} \cup I_{h}\right)$. Let $\Omega$ denote the domain,

\footnotetext{
*Corresponding author. E-mail: clicht@univ-montp2.fr.
} 
with a Lipschitz-continuous boundary $\partial \Omega$, occupied by the heterogeneous body under consideration. If $\varepsilon$ is a small number (the scaling parameter of the periodic structure), let $J_{\varepsilon}:=\left\{j \in \mathbb{Z}^{3} ; \varepsilon(j+Y) \subset \Omega\right\}$,

$$
\begin{array}{lll}
I_{\varepsilon}:=\bigcup_{j \in J_{\varepsilon}} \varepsilon(j+I), & S_{\varepsilon}:=\bigcup_{j \in J_{\varepsilon}} \varepsilon(j+S), & M_{\varepsilon}:=\Omega \backslash \bar{I}_{\varepsilon}, \\
I_{h, \varepsilon}:=\bigcup_{j \in J_{\varepsilon}} \varepsilon\left(j+I_{h}\right), & B_{h, \varepsilon}:=\bigcup_{j \in J_{\varepsilon}} \varepsilon\left(j+B_{h}\right), & M_{h, \varepsilon}:=\Omega \backslash\left(I_{h, \varepsilon} \cup \bar{B}_{h, \varepsilon}\right) .
\end{array}
$$

So in the case of two phases, $I_{\varepsilon}$ is the region occupied by the periodic distribution of inclusions, which does not intersect $\partial \Omega$, and $M_{\varepsilon}$ is the region occupied by the matrix. While in the three-phase case, $M_{h, \varepsilon}$, $B_{h, \varepsilon}, I_{h, \varepsilon}$ correspond to the regions occupied by the matrix, the interphase and inclusions, respectively.

To describe the constitutive equations of the body, we introduce $A_{M}, B_{M}$ in $L^{\infty}\left(M ; \operatorname{Lin}\left(\mathbb{S}^{3}\right)\right)$ and $A_{I}$, $B_{I}$ in $L^{\infty}\left(I ; \operatorname{Lin}\left(\mathbb{S}^{3}\right)\right)$, where $\operatorname{Lin}\left(\mathbb{S}^{3}\right)$ denotes the space of symmetric linear mappings from $\mathbb{S}^{3}$ to $\mathbb{S}^{3}$, the space of $3 \times 3$ symmetric matrices, with the usual inner product and norm denoted by $\cdot$ and || (as in $\mathbb{R}^{3}$ ). We assume that there exists $\kappa>0$ such that

$$
\begin{cases}A_{M}(y) \xi \cdot \xi, B_{M}(y) \xi \cdot \xi \geqslant \kappa|\xi|_{\mathbb{S}^{3}}^{2} & \text { a.e. } y \text { in } M, \forall \xi \in \mathbb{S}^{3} \\ A_{I}(y) \xi \cdot \xi, B_{I}(y) \xi \cdot \xi \geqslant \kappa|\xi|_{\mathbb{S}^{3}} & \text { a.e. } y \text { in } I, \forall \xi \in \mathbb{S}^{3}\end{cases}
$$

and extend these mappings into $\mathbb{R}^{3}$ by $Y$-periodicity. We will consider eight examples of composites indexed by $J$. The first six examples correspond to two phases occupying $M_{\varepsilon}$ and $I_{\varepsilon}$. In the first four examples, the constituents are assumed to be perfectly bonded along $S_{\varepsilon}$, whereas, in the last two examples, there are conditions of bilateral contact with Kelvin-Voigt type tangential friction

$$
\sigma_{T}=\mu_{e}^{*}[u]_{T}+\mu_{v}^{*}[\dot{u}]_{T}, \quad[u]_{N}=0 .
$$

Here $\mu_{e}^{*}, \mu_{v}^{*}$ are strictly positive real numbers and $[u]$ denotes the jump of displacement (the relative displacement) along $S_{\varepsilon}$, defined as the difference of the trace of the displacement $u$ in $I_{\varepsilon}$ with the one in $M_{\varepsilon}$ and $n, \sigma n$ being the inward normal to $\partial I_{\varepsilon}$ and the common stress vector along $S_{\varepsilon}$, respectively; one has

$$
[u]_{N}=[u] \cdot n, \quad[u]_{T}=[u]-[u]_{N} n, \quad \sigma_{N}=\sigma n \cdot n, \quad \sigma_{T}=\sigma n-\sigma_{N} n,
$$

while the upper $\operatorname{dot}^{\circ}$ stands for time derivative. In case $J=1$, the matrix and inclusions are assumed to be viscoelastic of Kelvin-Voigt type, with the elasticity and viscosity tensor equal to $A_{M}(x / \varepsilon)$, $B_{M}(x / \varepsilon)$ for all $x$ in $M_{\varepsilon}$, and $A_{I}(x / \varepsilon), B_{I}(x / \varepsilon)$ for all $x$ in $I_{\varepsilon}$; this is the case treated in [4]. Case $J=2$ corresponds to a viscoelastic matrix, with elasticity and viscosity tensors $A_{M}(x / \varepsilon)$ and $B_{M}(x / \varepsilon)$, surrounding linearly elastic inclusions of elasticity tensor $A_{I}(x / \varepsilon)$. Case $J=3$ is the "complement" of the previous one: viscoelastic inclusions with tensors $A_{I}(x / \varepsilon), B_{I}(x / \varepsilon)$ are periodically distributed in an elastic matrix with elasticity tensor $A_{M}(x / \varepsilon)$. Case $J=4$ deals with rigid inclusions in a viscoelastic matrix with tensors $A_{M}(x / \varepsilon), B_{M}(x / \varepsilon)$. Case $J=5$ corresponds to viscoelastic matrix and inclusions as when $J=1$, whereas $J=6$ corresponds to a purely linearly elastic matrix and inclusions with elasticity tensors $A_{M}(x / \varepsilon)$ and $A_{I}(x / \varepsilon)$, respectively. This last case with $\mu_{e}^{*}=0$ was treated by the asymptotic expansion method in [5]. Finally, we add two other examples involving three perfectly bonded phases occupying $M_{h, \varepsilon}, B_{h, \varepsilon}$ and $I_{h, \varepsilon}$. The interphase is assumed to be isotropically viscoelastic 
of Kelvin-Voigt type

$$
\sigma=\lambda_{e} \operatorname{tr} e(u) I_{\mathrm{d}}+2 \mu_{e} e(u)+\lambda_{v} \operatorname{tr} e(\dot{u}) I_{\mathrm{d}}+2 \mu_{v} e(\dot{u}),
$$

where $\lambda_{e}, \mu_{e}, \lambda_{v}, \mu_{v}$ are strictly positive numbers and $\sigma, e(u), e(\dot{u})$ denote stress, strain and strain rate tensors, and $I_{\mathrm{d}}$ stands for the identity matrix of $\mathbb{S}^{3}$. When $J=7$, matrix and inclusions are assumed to be viscoelastic with tensors $A_{M}(x / \varepsilon), B_{M}(x / \varepsilon), A_{I}(x / \varepsilon), B_{I}(x / \varepsilon)$, while if $J=8$, matrix and inclusions are purely linearly elastic with elasticity tensors $A_{M}(x / \varepsilon)$ and $A_{I}(x / \varepsilon)$, respectively.

Thus the problem of determining the quasi-static evolution during an interval of time $[0, T]$ of the body in a given initial state, clamped along $\Gamma_{0} \subset \partial \Omega$, with strictly positive two-dimensional Hausdorff measure $\mathcal{H}_{2}\left(\Gamma_{0}\right)$, and subjected to body forces in $\Omega$ and surface forces on $\Gamma_{1}=\partial \Omega \backslash \bar{\Gamma}_{0}$ of densities $f$, $g$, involves a set $s$ of data which reduces to only $\varepsilon$ in the first four examples, is $\left(\varepsilon, \mu_{e}^{*}, \mu_{v}^{*}\right)$ when $J=5,6$, and is $\left(\varepsilon, s^{\prime}\right), s^{\prime}=\left(h, \lambda_{e}, \mu_{e}, \lambda_{v}, \mu_{v}\right)$ in the last two examples. In the next section, we show that, under suitable data assumptions, the problem can be formulated as:

$$
\left\{\begin{array}{l}
\text { Find } u_{s} \text { in } C^{1}\left([0, T] ; H_{s}\right) \quad \text { such that } \\
a_{s}\left(u_{s}(t), \varphi\right)+b_{s}\left(\dot{u}_{s}(t), \varphi\right)=L(t)(\varphi) \quad \forall(\varphi, t) \in H_{s} \times[0, T] \\
u_{s}(0)=u_{s}^{o}
\end{array}\right.
$$

where $H_{s}$ is a Hilbert space of possible states with finite total strain energy equipped with the inner product $a_{s}(\cdot, \cdot) ; b_{s}$ is a continuous bilinear form on $H_{s}$ and $L(t)$ is a continuous linear form representing the work of the loading $(f, g)$ at time $t$. The existence and uniqueness of a field of displacement $u_{s}$ solution to $\left(P_{s}\right)$ is easily obtained by transforming $\left(P_{s}\right)$ in an evolution equation in $H_{s}$ and using the theory of semi-groups of linear operators. In Section 3, to determine the effective behavior of the composites we consider the geometrical and mechanical data $s$ as parameters taking values in a countable set and study the asymptotic behavior of $u_{s}$ when $\varepsilon$ goes to $0, \mu_{e}^{*}$ and $\mu_{v}^{*}$ go to infinity, $s^{\prime}$ goes to zero according to:

- $\exists\left(\bar{\mu}_{e}^{*}, \bar{\mu}_{v}^{*}\right) \in(0,+\infty)^{2} \quad$ such that $\lim \left(\mu_{e}^{*} \varepsilon, \mu_{v}^{*} \varepsilon\right)=\left(\bar{\mu}_{e}^{*}, \bar{\mu}_{v}^{*}\right)$,

- $\exists\left(\bar{\lambda}_{e}, \bar{\mu}_{e}, \bar{\lambda}_{v}, \bar{\mu}_{v}\right) \in(0,+\infty)^{4}$ such that

$$
\lim \left(\lambda_{e} / 2 h, \mu_{e} / 2 h, \lambda_{v} / 2 h, \mu_{v} / 2 h\right)=\left(\bar{\lambda}_{e}, \bar{\mu}_{e}, \bar{\lambda}_{v}, \bar{\mu}_{v}\right), \quad \overline{\lim } \varepsilon^{2} / \mu_{e}=\varlimsup \lim \varepsilon^{2} / h=0 .
$$

Note that $s^{\prime}$ and $\varepsilon$ go quite independently to zero under the sole condition $\varlimsup \varepsilon^{2} / \mu_{e}=\varlimsup \lim \varepsilon^{2} / h=0$ which means that the Lamé coefficient $\mu_{e}$ and the thickness of the interphase are not too small. In the following, we will say that $s$ goes to $\bar{s}$ according to (1.3).

By using the Laplace transform and a suitable assumption on the initial state, it can be shown that in all examples the effective constitutive equation is of the type

$$
\sigma=A^{\mathrm{eff}} e(u)+B^{\mathrm{eff}} e(\dot{u})+\int_{0}^{t} K(t-\tau) e(\dot{u})(\tau) \mathrm{d} \tau
$$

where $A^{\text {eff }}$ and $B^{\text {eff }}$ are the standard homogenized tensors corresponding to the heterogeneous elastic and viscous behaviors, while the kernel $K$ is a perfectly identified element of $\operatorname{Lin}\left(\mathbb{S}^{3}\right)$. In some cases $B^{\text {eff }}$ may vanish.

The principle of this study is that of [4] but due to the character, say, singular of the cases $J \neq 1$ (purely elastic behavior in some regions and/or soft thin interphase, or viscoelastic friction on the interfaces) 
which are relevant in practice, there are some technical difficulties that must be overcome $\left(H_{s}\right.$ may be strongly $s$-dependent, $b_{s}$ may have a kernel and consequently $\left(P_{s}\right)$ involves an unbounded evolution operator and convergences do occur in weak-* topologies).

\section{A result of existence and uniqueness for $\left(P_{s}\right)$}

We define the space $H_{s}$ as follows:

$$
\begin{cases}1 \leqslant J \leqslant 3 & H_{s}:=H_{\Gamma_{0}}^{1}\left(\Omega ; \mathbb{R}^{3}\right):=\left\{u \in H^{1}\left(\Omega ; \mathbb{R}^{3}\right) ; u=0 \text { on } \Gamma_{0}\right\} \\ J=4 & H_{s}:=\left\{u \in H_{\Gamma_{0}}^{1}\left(\Omega ; \mathbb{R}^{3}\right) ; e(u)=0 \text { in } I_{\varepsilon}\right\}, \\ & \text { with } e \text { being the symmetrized gradient in the sense of distributions, } \\ J=5,6 & H_{s}:=\left\{u \in H_{\Gamma_{0}}^{1}\left(\Omega \backslash S_{\varepsilon} ; \mathbb{R}^{3}\right) ;[u]_{N}=0 \text { on } S_{\varepsilon}\right\} \\ J=7,8 & H_{s}:=H_{\Gamma_{0}}^{1}\left(\Omega ; \mathbb{R}^{3}\right),\end{cases}
$$

it is equipped with the inner product, equivalent to the classical one, associated with the bilinear form $a_{s}$ defined by:

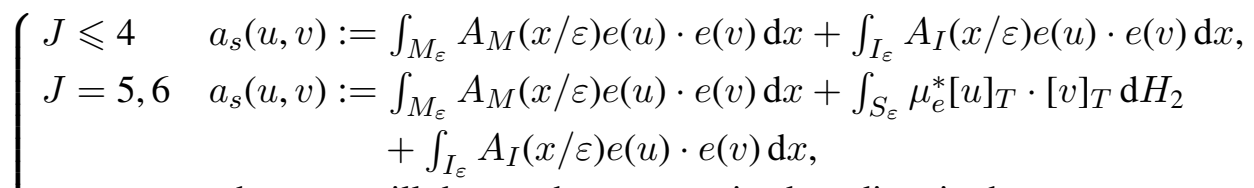

$$
\begin{aligned}
& \text { where we still denote the symmetrized gradient in the } \\
& \text { sense of distribution in } \Omega \backslash S_{\varepsilon} \text { by } e \text {, } \\
& J=7,8 \quad a_{s}(u, v):=\int_{M_{h, \varepsilon}} A_{M}(x / \varepsilon) e(u) \cdot e(v) \mathrm{d} x \\
& +\int_{B_{h, \varepsilon}}\left(\lambda_{e} \operatorname{tr} e(u) \operatorname{tr} e(v)+2 \mu_{e} e(u) \cdot e(v)\right) \mathrm{d} x \\
& +\int_{I_{h, \varepsilon}} A_{I}(x / \varepsilon) e(u) \cdot e(v) \mathrm{d} x,
\end{aligned}
$$

Let $b_{s}$ be the continuous bilinear form on $H_{s}$ deduced from the irreversible part of the behavior of the body

$$
\left\{\begin{array}{rlrl}
J=1 & b_{s}(u, v):= & \int_{M_{\varepsilon}} B_{M}(x / \varepsilon) e(u) \cdot e(v) \mathrm{d} x+\int_{I_{\varepsilon}} B_{I}(x / \varepsilon) e(u) \cdot e(v) \mathrm{d} x \\
J=2,4 \quad & b_{s}(u, v):= & \int_{M_{\varepsilon}} B_{M}(x / \varepsilon) e(u) \cdot e(v) \mathrm{d} x \\
J=3 & b_{s}(u, v):= & \int_{I_{\varepsilon}} B_{I}(x / \varepsilon) e(u) \cdot e(v) \mathrm{d} x \\
J=5 & b_{s}(u, v):= & \int_{M_{\varepsilon}} B_{M}(x / \varepsilon) e(u) \cdot e(v) \mathrm{d} x+\int_{S_{\varepsilon}} \mu_{v}^{*}[u]_{T} \cdot[v]_{T} \mathrm{~d} H_{2} \\
& +\int_{I_{\varepsilon}} B_{I}(x / \varepsilon) e(u) \cdot e(v) \mathrm{d} x \\
J=6 & & b_{s}(u, v):= & \int_{S_{\varepsilon}} \mu_{v}^{*}[u]_{T} \cdot[v]_{T} \mathrm{~d} H_{2} \\
J=7 & b_{s}(u, v):= & \int_{M_{h, \varepsilon}} B_{M}(x / \varepsilon) e(u) \cdot e(v) \mathrm{d} x \\
& +\int_{B_{h, \varepsilon}}\left(\lambda_{v} \operatorname{tr} e(u) \operatorname{tr} e(v)+2 \mu_{v} e(u) \cdot e(v)\right) \mathrm{d} x \\
& & +\int_{I_{h, \varepsilon}} B_{I}(x / \varepsilon) e(u) \cdot e(v) \mathrm{d} x \\
& & & \\
J=8 & b_{s}(u, v):= & \int_{B_{h, \varepsilon}}\left(\lambda_{v} \operatorname{tr} e(u) \operatorname{tr} e(v)+2 \mu_{v} e(u) \cdot e(v)\right) \mathrm{d} x
\end{array}\right.
$$


If the loading $(f, g)$ is assumed to be in $C^{0}\left([0, T] ; L^{2}\left(\Omega ; \mathbb{R}^{3}\right) \times L^{2}\left(\Gamma_{1} ; \mathbb{R}^{3}\right)\right)$, the quasistatic evolution of the body in initial state $u_{s}^{o}$ in $H_{s}$ may be formulated as:

$$
\left\{\begin{array}{l}
\text { Find } u_{s} \text { in } C^{1}\left([0, T] ; H_{s}\right) \quad \text { such that } \\
a_{s}\left(u_{s}(t), v\right)+b_{s}\left(\dot{u}_{s}(t), v\right)=L(t)(v) \quad \forall(v, t) \in H_{s} \times[0, T] \\
u_{s}(0)=u_{s}^{o}
\end{array}\right.
$$

where

$$
L(t)(v):=\int_{\Omega} f(x, t) \cdot v(x) \mathrm{d} x+\int_{\Gamma_{1}} g(x, t) \cdot v(x) \mathrm{d} H_{2} \quad \forall(v, t) \in H_{s} \times[0, T] .
$$

To solve $\left(P_{s}\right)$, we make the additional assumption

$$
(f, g) \in C^{1, \alpha}\left([0, T] ; L^{2}\left(\Omega ; \mathbb{R}^{3}\right) \times L^{2}\left(\Gamma_{1} ; \mathbb{R}^{3}\right)\right), \quad \alpha \in(0,1],
$$

and seek $u_{s}$ in the form

$$
u_{s}=u_{s}^{e}+u_{s}^{r},
$$

where $u_{s}^{e}$ satisfies

$$
\exists ! u_{s}^{e}(t) \in C^{1, \alpha}\left([0, T] ; H_{s}\right) ; \quad a_{s}\left(u_{s}^{e}(t), v\right)=L(t) v \quad \forall(v, t) \in H_{s} \times[0, T] .
$$

Let

$$
\left\{\begin{array}{l}
\operatorname{ker} b_{s}:=\left\{u \in H_{s} ; b_{s}(u, v)=0 \forall v \in H_{s}\right\}, \\
V_{s}:=\operatorname{ker} b_{s}^{\perp}:=\left\{u \in H_{s} ; a_{s}(u, v)=0 \forall v \in \operatorname{ker} b_{s}\right\},
\end{array}\right.
$$

then the remaining part $u_{s}^{r}$ will therefore be involved in an evolution equation in $V_{s}$ governed by the following operator:

$$
\left\{\begin{array}{l}
D\left(A_{s}\right)=\left\{u \in V_{s} ; \exists ! w(u) \in V_{s} \text { s.t. } b_{s}(w(u), v)+a_{s}(u, v)=0 \forall v \in V_{s}\right\}, \\
A_{s} u=w(u) .
\end{array}\right.
$$

Proposition 2.1. $A_{s}$ is an m-dissipative operator.

\section{Proof.}

(i) $A_{s}$ is dissipative because, for all $u$ in $D\left(A_{s}\right)$, we have

$$
a_{s}\left(A_{s} u, u\right)=a_{s}(w(u), u)=-b_{s}(w(u), w(u)) \leqslant 0 .
$$

(ii) $I-A_{s}$ is onto.

Let $F$ be in $V_{s}$, if $u-A_{s} u=F$, we should have

$$
u \in V_{s} ; \quad b_{s}(u-F, v)+a_{s}(u, v)=0 \quad \forall v \in V_{s},
$$

which has a unique solution $\bar{u}$ by the Lax-Milgram lemma. Hence, $\bar{u} \in D\left(A_{s}\right)$ and $\bar{u}-A_{s} \bar{u}=F$. 
(iii) $A_{s}$ is self-adjoint: as $A_{s}$ is $m$-dissipative, it suffices to prove that $A_{s}$ is symmetric, which is true because for all $u, v$ in $D\left(A_{s}\right)$ one has

$$
a_{s}\left(A_{s} u, v\right)=a_{s}(w(u), v)=-b_{s}(w(u), w(v))=a_{s}(u, w(v))=a_{s}\left(u, A_{s} v\right) .
$$

Lastly, it is straight forward to check that $\left(P_{s}\right)$ is equivalent to

$$
\left\{\begin{array}{l}
\frac{\mathrm{d} u_{s}^{r}}{\mathrm{~d} t}=A_{s} u_{s}^{r}-\frac{\mathrm{d}\left(u_{s}^{e}\right)^{\perp}}{\mathrm{d} t}, \\
u_{s}^{r}(0)=u_{s}^{o}-u_{s}^{e}(0):=u_{s}^{r o},
\end{array}\right.
$$

where $u_{s}^{e \perp}$ denotes the projection of $u_{s}^{e}$ on $V_{s}$, which classically has a unique solution if $u_{s}^{r o} \in$ $u_{s}^{e}(0)+D\left(A_{s}\right)$. Hence the following result is established.

Theorem 2.1. If $(f, g) \in C^{1, \alpha}\left([0, T] ; L^{2}\left(\Omega ; \mathbb{R}^{3}\right) \times L^{2}\left(\Gamma_{1} ; \mathbb{R}^{3}\right)\right)$ for some $\alpha$ in $(0,1]$, then $\left(P_{s}\right)$ has a unique solution of class $C^{1}\left([0, T] ; H_{s}\right)$ when $u_{s}^{o} \in u_{s}^{e}(0)+D\left(A_{s}\right)$, of class $C^{0}\left([0, T] ; H_{s}\right) \cap$ $C^{1}\left((0, T] ; H_{s}\right)$ when $u_{s}^{o} \in u_{s}^{e}(0)+V_{s}$.

Remark 2.1. If the kernel of $b_{s}$ is not reduced to $\{0\}$, the operator $A_{s}$ is unbounded. In the other cases $(J \in\{1,4,5,7\})$, by equipping $H_{s}$ with $b_{s}$ as inner product (like in [4] for $J=1$ ), one may formulate $\left(P_{s}\right)$ in terms of an ordinary differential equation, and the conclusions of Theorem 2.1 are reached just with the milder assumption $(f, g) \in C^{0}\left([0, T] ; L^{2}\left(\Omega ; \mathbb{R}^{3}\right) \times L^{2}\left(\Gamma_{1} ; \mathbb{R}^{3}\right)\right)$.

\section{Effective behavior of the body}

As previously stated, the strategy is the one of [4]. By using the Laplace transform and a suitable assumption on the initial state, the problem reduces to two problems of homogenization in elasticity. In the non-standard cases $(J \geqslant 7)$, we solve them by a variational convergence method (see [2,3]), and the obtained "homogenized coefficients" expressions permit us to determine the effective behavior of the heterogeneous viscoelastic body. We proceed in five steps, and, as usual, $C$ and $C^{\prime}$ will denote various constants.

First step (Extension of $u_{s}$ into $\left.[0,+\infty)\right)$. Let $(\tilde{f}, \tilde{g})$ be a $C^{1, \alpha}\left([0,+\infty) ; L^{2}\left(\Omega ; \mathbb{R}^{3}\right) \times L^{2}\left(\Gamma_{1} ; \mathbb{R}^{3}\right)\right)$ extension of $(f, g)$ with compact support in $[0, T+1)$, then the solution $u_{s}$ to $\left(P_{s}\right)$ can be viewed as the restriction to $[0, T]$ of the unique solution $\tilde{u}_{s}$ to

$$
\left\{\begin{array}{l}
a_{s}\left(\tilde{u}_{s}, v\right)+b_{s}\left(\dot{\tilde{u}}_{s}, v\right)=\int_{\Omega} \tilde{f} \cdot v \mathrm{~d} x+\int_{\Gamma_{1}} \tilde{g} \cdot v \mathrm{~d} H_{2} \quad \forall(v, t) \in H_{s} \times[0,+\infty), \\
\tilde{u}_{s}(0)=u_{s}^{o} \in u_{s}^{e}(0)+V_{s}
\end{array}\right.
$$

which definitely exists in $L^{\infty}\left(0,+\infty ; H_{s}\right) \cap C^{1}\left([0,+\infty) ; H_{s}\right)$ when $J \in\{1,4,5,7\}$ (see Remark 2.1) and in $L^{\infty}\left(0,+\infty ; H_{s}\right) \cap C^{0}\left([0,+\infty) ; H_{s}\right) \cap C^{1}\left((0,+\infty) ; H_{s}\right)$ when $J \in\{2,3,6,8\}$. The inequality

$$
a_{s}(u, u) \geqslant \kappa|e(u)|_{L^{2}\left(\Omega ; \mathbb{S}^{3}\right)}^{2} \quad \forall u \in H_{s}, J \in\{1,2,3,4\},
$$


due to (1.1), does not hold when $J \in\{5,6,7,8\}$, but one has

$$
a_{s}(u, u) \geqslant C\left(|u|_{L^{2}\left(\Omega ; \mathbb{R}^{3}\right)}^{2}+|u|_{B D(\Omega)}^{2}\right) \quad \forall u \in H_{s}, J \in\{5,6,7,8\},
$$

where we recall that

$$
\begin{aligned}
& B D(\Omega):=\left\{u \in L^{1}\left(\Omega ; \mathbb{R}^{3}\right) ; e(u) \in M_{b}\left(\Omega ; \mathbb{S}^{3}\right)\right\}, \\
& M_{b}\left(\Omega ; \mathbb{S}^{3}\right):=\left\{\text { bounded } \mathbb{S}^{3} \text {-valued measures on } \Omega\right\}, \\
& L D(\Omega):=\left\{u \in L^{1}\left(\Omega ; \mathbb{R}^{3}\right) ; e(u) \in L^{1}\left(\Omega ; \mathbb{S}^{3}\right)\right\} .
\end{aligned}
$$

Indeed, if $J \in\{7,8\}$, one has

$$
\begin{aligned}
a_{s}(u, u) & \geqslant \kappa|e(u)|_{L^{2}\left(M_{h, \varepsilon} \cup I_{h, \varepsilon} ; \mathbb{S}^{3}\right)}^{2}+\mu_{e}|e(u)|_{L^{2}\left(B_{h, \varepsilon} ; \mathbb{S}^{3}\right)}^{2} \\
& \geqslant(\kappa /|\Omega|)|e(u)|_{L^{1}\left(M_{h, \varepsilon} \cup I_{h, \varepsilon} ; \mathbb{S}^{3}\right)}^{2}+\left(C \mu_{e} / h\right)|e(u)|_{L^{1}\left(B_{h, \varepsilon} ; \mathbb{S}^{3}\right)}^{2} \\
& \geqslant C|e(u)|_{L^{1}\left(\Omega ; \mathbb{S}^{3}\right)}^{2} \geqslant C|u|_{L D(\Omega)}^{2}=C|u|_{B D(\Omega)}^{2}
\end{aligned}
$$

because $u$ vanishes on $\Gamma_{0}$, see [12]. To complete the proof of (3.3), we first recall that, using a variant of [11], p. 183, involving rigid displacements, any $u$ in $H_{\Gamma_{0}}^{1}\left(M_{h_{o}, \varepsilon} ; \mathbb{R}^{3}\right)$ has an extension $\tilde{u}$ into $H_{\Gamma_{0}}^{1}\left(\Omega ; \mathbb{R}^{3}\right)$ such that $|e(\tilde{u})|_{L^{2}\left(\Omega ; \mathbb{S}^{3}\right)} \leqslant C|e(u)|_{L^{2}\left(M_{h_{o},} ; \mathbb{R}^{3}\right)}$, where $h_{o}$ is a fixed positive number less than $\operatorname{dist}(S, \partial Y)$. Thus the Korn inequality in $H_{\Gamma_{0}}^{1}\left(\Omega ; \mathbb{R}^{3}\right)$ yields

$$
|\tilde{u}|_{L^{2}\left(\Omega ; \mathbb{R}^{3}\right)}^{2} \leqslant C|e(\tilde{u})|_{L^{2}\left(\Omega ; \mathbb{S}^{3}\right)}^{2} \leqslant C^{\prime}|e(u)|_{L^{2}\left(M_{h_{o}, \varepsilon} ; \mathbb{S}^{3}\right)}^{2} \leqslant\left(C^{\prime} / \kappa\right) a_{s}(u, u) .
$$

Next the periodic structure of $M_{h_{o}, \varepsilon}$ and the Korn inequality in $H_{\Gamma_{0}}^{1}\left(\Omega \backslash M_{h_{o}, \varepsilon}\right)$ give

$$
\begin{aligned}
|u-\tilde{u}|_{L^{2}\left(\Omega ; \mathbb{R}^{3}\right)}^{2} & =|u-\tilde{u}|_{L^{2}\left(\Omega \backslash M_{h_{o}, \varepsilon} ; \mathbb{R}^{3}\right)}^{2} \leqslant C \varepsilon^{2}|e(u-\tilde{u})|_{L^{2}\left(\Omega \backslash M_{h_{o}, \varepsilon} ; \mathbb{S}^{3}\right)}^{2} \\
& \leqslant C \varepsilon^{2}\left(|e(\tilde{u})|_{L^{2}\left(\Omega ; \mathbb{S}^{3}\right)}^{2}+|e(u)|_{L^{2}\left(M_{h, \varepsilon} ; \mathbb{S}^{3}\right)}^{2}+|e(u)|_{L^{2}\left(B_{h, \varepsilon} ; \mathbb{S}^{3}\right)}^{2}+|e(u)|_{L^{2}\left(I_{h, \varepsilon} ; \mathbb{S}^{3}\right)}^{2}\right) \\
& \leqslant C \varepsilon^{2}\left(1+\frac{1}{\mu_{e}}\right) a_{s}(u, u) .
\end{aligned}
$$

Then (3.4) implies

$$
|u|_{L^{2}\left(\Omega ; \mathbb{R}^{3}\right)}^{2} \leqslant C\left(1+\varepsilon^{2}+\frac{\varepsilon^{2}}{\mu_{e}}\right) a_{s}(u, u)
$$

which, with assumption (1.3), achieves the proof of (3.3).

Remark 3.1. The weaker assumption $\overline{\lim } \varepsilon^{2} / \mu_{e}<+\infty$ is enough to get (3.3). But, as we shall see in the proof of Proposition 3.1, it is crucial to know that a sequence $\left(u_{s}\right)$ such that $a_{s}\left(u_{s}, u_{s}\right) \leqslant C$ 
is strongly relatively compact in $L^{2}\left(\Omega ; \mathbb{R}^{3}\right)$ and that any limit point belongs to $H_{\Gamma_{0}}^{1}\left(\Omega ; \mathbb{R}^{3}\right)$. Actually, (3.4) implies that $\left(\tilde{u}_{s}\right)$ is weakly relatively compact in $H_{\Gamma_{0}}^{1}\left(\Omega ; \mathbb{R}^{3}\right)$ and, by Rellich Theorem, strongly relatively compact in $L^{2}\left(\Omega ; \mathbb{R}^{3}\right)$, then (3.5) with assumption (1.3) yield the strong relative compactness in $L^{2}\left(\Omega ; \mathbb{R}^{3}\right)$ of $\left(u_{s}\right)$ and that any limit point belongs to $H_{\Gamma_{0}}^{1}\left(\Omega ; \mathbb{R}^{3}\right)$.

In the case $J \in\{5,6\}$ the same argument with $h_{o}=0$ (see [5]) gives

$$
|u|_{L^{2}\left(\Omega ; \mathbb{R}^{3}\right)}^{2} \leqslant C a_{s}(u, u) \quad \forall u \in H_{s}
$$

To conclude, it should be noted, that for all $\tau$ in $C_{0}^{0}\left(\bar{\Omega} ; \mathbb{S}^{3}\right) \cap C^{1}\left(\Omega ; \mathbb{S}^{3}\right)$ we have

$$
\begin{aligned}
-\int_{\Omega} u \cdot \operatorname{div} \tau \mathrm{d} x & =-\int_{M_{\varepsilon}} u \cdot \operatorname{div} \tau \mathrm{d} x-\int_{I_{\varepsilon}} u \cdot \operatorname{div} \tau \mathrm{d} x \\
& =\int_{M_{\varepsilon}} e(u) \cdot \tau \mathrm{d} x+\int_{I_{\varepsilon}} e(u) \cdot \tau \mathrm{d} x-\int_{S_{\varepsilon}} \tau n[u] \mathrm{d} H_{2} \\
& \leqslant C|\tau|_{C^{0}\left(\bar{\Omega} ; \mathbb{S}^{3}\right)} a_{s}(u, u)^{1 / 2},
\end{aligned}
$$

where $C_{0}^{0}\left(\bar{\Omega} ; \mathbb{S}^{3}\right):=\left\{\tau \in C^{0}\left(\bar{\Omega} ; \mathbb{S}^{3}\right) ; \tau=0\right.$ on $\left.\partial \Omega\right\}$. Thus, (3.1)-(3.3) imply

$$
\left\{\begin{array}{l}
\text { when } J \in\{1,4\} \tilde{u}_{s} \text { is bounded in } W^{1, \infty}\left(0,+\infty ; H_{\Gamma_{0}}^{1}\left(\Omega ; \mathbb{R}^{3}\right)\right), \\
\text { when } J \in\{2,3\} \tilde{u}_{s} \text { is bounded in } L^{\infty}\left(0,+\infty ; H_{\Gamma_{0}}^{1}\left(\Omega ; \mathbb{R}^{3}\right)\right) \\
\text { when } J \in\{5,7\} \tilde{u}_{s} \text { is bounded in } W^{1, \infty}\left(0,+\infty ; B D(\Omega) \cap L^{2}\left(\Omega ; S^{3}\right)\right) \\
\text { when } J \in\{6,8\} \tilde{u}_{s} \text { is bounded in } L^{\infty}\left(0,+\infty ; B D(\Omega) \cap L^{2}\left(\Omega ; S^{3}\right)\right)
\end{array}\right.
$$

Second step (Laplace transform of $\left(P_{s}\right)$ ). So, if for any Banach space $X, \mathcal{L} z$ denotes the Laplace transform

$$
\mathcal{L} z(p):=\int_{0}^{+\infty} \exp (-p t) z(t) \mathrm{d} t \quad \forall p \in(0,+\infty)
$$

of any function of $L^{\infty}(0,+\infty ; X)$, one has for all $p \in(0,+\infty)$

$$
\left\{\begin{array}{l}
\mathcal{L} u_{s}(p) \in H_{s} \\
\left(a_{s}+p b_{s}\right)\left(\mathcal{L} u_{s}(p), v\right)=b_{s}\left(u_{s}^{o}, v\right)+\int_{\Omega} \mathcal{L} \tilde{f} \cdot v \mathrm{~d} x+\int_{\Gamma_{1}} \mathcal{L} \tilde{g} \cdot v \mathrm{~d} H_{2} \quad \forall v \in H_{s}
\end{array}\right.
$$

Similar to [4], one makes the fundamental assumption of admissibility for the initial state

$$
\left\{\begin{array}{l}
\exists\left(f^{o}, g^{o}\right) \in L^{2}\left(\Omega ; \mathbb{R}^{3}\right) \times L^{2}\left(\Gamma_{1} ; \mathbb{R}^{3}\right) \quad \text { such that } \\
a_{s}\left(u_{s}^{o}, v\right)=\int_{\Omega} f^{o} \cdot v \mathrm{~d} x+\int_{\Gamma_{1}} g^{o} \cdot v \mathrm{~d} H_{2} \quad \forall v \in H_{s}
\end{array}\right.
$$


Hence (3.9) can be written

$$
\left\{\begin{array}{l}
\theta_{s}:=p \mathcal{L} u_{s}(p)-u_{s}^{o} \in H_{s} \\
\left(a_{s} / p+b_{s}\right)\left(\theta_{s}, v\right) \\
\quad=\int_{\Omega} \mathcal{L} \tilde{f} \cdot v \mathrm{~d} x+\int_{\Gamma_{1}} \mathcal{L} \tilde{g} \cdot v \mathrm{~d} H_{2}-\frac{1}{p}\left(\int_{\Omega} f^{o} \cdot v \mathrm{~d} x+\int_{\Gamma_{1}} g^{o} \cdot v \mathrm{~d} H_{2}\right) \quad \forall v \in H_{s},
\end{array}\right.
$$

so that the study of the asymptotic behavior of $u_{s}$ reduces to two problems of homogenization in linearized elasticity involving the bilinear forms $a_{s}$ and $c_{s}(p):=a_{s} / p+b_{s}$, respectively.

Third step (Convergence of $\theta_{s}$ and $u_{s}^{o}$ ). Cases $J \leqslant 3$ are classical (see [10]), while cases $4 \leqslant J \leqslant 6$ were treated in [5]. The other cases seem new and the proof of the homogenization result is given in Section 4. We now need to introduce some tools. If

$$
\left\{\begin{array}{l}
H_{\mathrm{per}}^{1}\left(Y ; \mathbb{R}^{3}\right):=\left\{u \in H^{1}\left(Y ; \mathbb{R}^{3}\right) ; u \text { takes the same values on the opposite sides of } \partial Y\right\}, \\
H_{\mathrm{per}}^{1}\left(Y \backslash S ; \mathbb{R}^{3}\right) \\
\quad:=\left\{u \in H^{1}\left(Y \backslash S ; \mathbb{R}^{3}\right) ; u \text { takes the same values on the opposite sides of } \partial Y\right\},
\end{array}\right.
$$

on the space

$$
H_{Y}=\left\{u \in H_{\text {per }}^{1}\left(Y \backslash S, \mathbb{R}^{3}\right) ;[u]_{N}=0 \text { on } S\right\}, \quad \text { when } J \in\{5,6\},
$$

are defined continuous bilinear forms $a_{Y}, b_{Y}$ whose expressions are derived from those of $a_{s}, b_{s}$ by replacing $M_{\varepsilon}, I_{\varepsilon}, S_{\varepsilon}$ and $x / \varepsilon$ by $M, I, S$ and $y$ the current point of the unit cell $Y$ which describes the $\varepsilon Y$-periodic structure of the body. For instance, $a_{Y}(\varphi, \psi)$ reads as

$$
a_{Y}(\varphi, \psi):=\int_{M} A_{M}(y) e(\varphi) \cdot e(\psi) \mathrm{d} y+\int_{S} \mu^{*}[\varphi]_{T} \cdot[\psi]_{T} \mathrm{~d} H_{2}+\int_{I} A_{I}(y) e(\varphi) \cdot e(\psi) \mathrm{d} y .
$$

For $J \in\{7,8\}$, we have

$$
H_{Y}=H_{\text {per }}^{1}\left(Y \backslash S ; \mathbb{R}^{3}\right),
$$

and the bilinear form $a_{Y}$ is defined by

$$
\begin{aligned}
a_{Y}(\varphi, \psi)= & \int_{M} A_{M}(y) e(\varphi) \cdot e(\psi) \mathrm{d} y \\
& +\int_{S}\left(\bar{\lambda}_{e}[\varphi]_{N}[\psi]_{N}+2 \bar{\mu}_{e}\left([\varphi] \otimes_{S} n\right) \cdot\left([\psi] \otimes_{S} n\right)\right) \mathrm{d} H_{2} \\
& +\int_{I} A_{I}(y) e(\varphi) \cdot e(\psi) \mathrm{d} y \quad \forall \varphi, \psi \in H_{Y}
\end{aligned}
$$

while the continuous bilinear form $b_{Y}$ on $H_{Y}$ is defined by

$$
b_{Y}(\varphi, \psi)=\int_{M} B_{M}(y) e(\varphi) \cdot e(\psi) \mathrm{d} y
$$




$$
\begin{aligned}
& +\int_{S}\left(\bar{\lambda}_{v}[\varphi]_{N}[\psi]_{N}+2 \bar{\mu}_{v}\left([\varphi] \otimes_{S} n\right) \cdot\left([\psi] \otimes_{S} n\right)\right) \mathrm{d} H_{2} \\
& +\int_{I} B_{I}(y) e(\varphi) \cdot e(\psi) \mathrm{d} y \quad \forall \varphi, \psi \in H_{Y}
\end{aligned}
$$

when $J=7$, whilst when $J=8$ only the surface term appears, where $\xi \otimes_{S} \zeta$ is the symmetrized tensor product of $\xi$ and $\zeta$ in $\mathbb{R}^{3}$.

Let

$$
c_{Y}(p):=\frac{1}{p} a_{Y}+b_{Y}
$$

as $a_{Y}$ is coercive on $H_{Y} / \mathbb{R}^{3}$, we deduce that for all $E$ in $\mathbb{S}^{3}$ the problems

$$
\begin{aligned}
& \min \left\{\frac{1}{2} a_{Y}(E \cdot+v, E \cdot+v), v \in H_{Y}\right\}, \\
& \min \left\{\frac{1}{2} c_{Y}(p)(E \cdot+v, E \cdot+v), v \in H_{Y}\right\},
\end{aligned}
$$

where $E$. denotes the function $y \in Y \backslash S \mapsto E y \in \mathbb{R}^{3}$, have unique solutions denoted by $v_{E}^{a}, v_{E}^{c(p)}$, respectively, and satisfying

$$
\begin{cases}a_{Y}\left(v_{E}^{a}, \varphi\right)=-a_{Y}(E \cdot, \varphi) & \forall \varphi \in H_{Y}, \\ c_{Y}(p)\left(v_{E}^{c(p)}, \varphi\right)=-c_{Y}(p)(E \cdot, \varphi) & \forall \varphi \in H_{Y}, \\ \int_{Y} v_{E}^{a}(y) \mathrm{d} y=\int_{Y} v_{E}^{c(p)}(y) \mathrm{d} y=0 . & \end{cases}
$$

Let $A^{\text {eff }}$ and $C^{\text {eff }}(p)$ be the elements of $\operatorname{Lin}\left(\mathbb{S}^{3}\right)$ defined by

$$
\begin{cases}A^{\mathrm{eff}} E \cdot E:=a_{Y}\left(E \cdot+v_{E}^{a}, E \cdot+v_{E}^{a}\right) & \forall E \in \mathbb{S}^{3}, \\ C^{\mathrm{eff}}(p) E \cdot E:=c_{Y}(p)\left(E \cdot+v_{E}^{c(p)}, E \cdot+v_{E}^{c(p)}\right) & \forall E \in \mathbb{S}^{3},\end{cases}
$$

which classically satisfy:

$$
\begin{array}{llll}
\exists \bar{\kappa}_{M} ; & \left|A^{\mathrm{eff}} E\right| \leqslant \bar{\kappa}_{M}|E|, \quad & \left|C^{\mathrm{eff}}(p) E\right| \leqslant \bar{\kappa}_{M}|E| & \forall E \in \mathbb{S}^{3} \\
\exists \bar{\kappa}_{m} ; & A^{\mathrm{eff}} E \cdot E \geqslant \bar{\kappa}_{m}|E|^{2}, & C^{\mathrm{eff}}(p) E \cdot E \geqslant \bar{\kappa}_{m}|E|^{2} & \forall E \in \mathbb{S}^{3} .
\end{array}
$$

Then the following convergence result, which has its own interest (homogenization and bonding in linearized elasticity) and whose proof is given Section 4 by a method of variational convergence, holds. 
Proposition 3.1. For all $p$ in $(0,+\infty)$, when $s$ goes to $\bar{s}$ according to $(1.3),\left(u_{s}^{o}, \theta_{s}\right)$ converges weakly in $H^{1}\left(\Omega ; \mathbb{R}^{3}\right)$ when $J \leqslant 4$, weak-* in $B D(\Omega)$ and strongly in $L^{2}\left(\Omega ; \mathbb{R}^{3}\right)$ when $J \geqslant 5$, toward the unique solution $\left(\bar{u}^{o}, \bar{\theta}\right)$ to

$$
\left\{\begin{array}{l}
\left(\bar{u}^{o}, \bar{\theta}\right) \in H_{\Gamma_{0}}^{1}\left(\Omega ; \mathbb{R}^{3}\right) \\
\int_{\Omega} A^{\mathrm{eff}} e\left(\bar{u}^{o}\right) \cdot e(v) \mathrm{d} x=\int_{\Omega} f^{o} \cdot v \mathrm{~d} x+\int_{\Gamma_{1}} g^{o} \cdot v \mathrm{~d} H_{2} \\
\int_{\Omega} C^{\mathrm{eff}}(p) e(\bar{\theta}) \cdot e(v) \mathrm{d} x \\
\quad=\int_{\Omega} \mathcal{L} \tilde{f} \cdot \tilde{v} \mathrm{~d} x+\int_{\Gamma_{1}} \mathcal{L} \tilde{g} \cdot v \mathrm{~d} H_{2}-\frac{1}{p}\left(\int_{\Omega} f^{o} \cdot v \mathrm{~d} x+\int_{\Gamma_{1}} g^{o} \cdot v \mathrm{~d} H_{2}\right) \quad \forall v \in H_{\Gamma_{0}}^{1}\left(\Omega ; \mathbb{R}^{3}\right) .
\end{array}\right.
$$

Fourth step (A fundamental identity). In order to more thoroughly identify the asymptotic behavior of $u_{s}$, we establish a fundamental identity satisfied by $C^{\text {eff }}(p)$ in terms of the effective tensor of elasticity $A^{\text {eff }}$ and the "effective tensor of viscosity" $B^{\text {eff }}$ defined as follows. Let

$$
\begin{aligned}
& \operatorname{ker} b_{Y}:=\left\{\varphi \in H_{Y} ; b_{Y}(\varphi, \psi)=0 \forall \psi \in H_{Y}\right\}, \\
& V_{Y}:=\operatorname{ker} b_{Y}^{\perp}:=\left\{\varphi \in H_{Y} ; a_{Y}(\varphi, \psi)=0 \forall \psi \in \operatorname{ker} b_{Y}\right\} .
\end{aligned}
$$

Actually, the closed affine manifold

$$
C_{Y}:=\left\{\varphi \in H_{Y} ; b_{Y}(\varphi, \psi)=-b_{Y}(E \cdot, \psi) \forall \psi \in H_{Y}\right\}
$$

is not empty. This is obvious when $J \in\{1,4,5,7\}$ due to the coercivity of $b_{Y}$ on $H_{Y}$. When $J=3$, any $H^{1}$-extension of $-E$. into $M$ which is $Y$-periodic belongs to $C_{Y}$. When $J=2, b_{Y}$ is coercive on $H_{\text {per }}^{1}\left(M ; \mathbb{R}^{3}\right)=\left\{\psi \in H^{1}\left(M ; \mathbb{R}^{3}\right) ; \psi\right.$ takes the same values on opposite sides of $\left.\partial Y\right\}$, then any $H^{1}$ extension into $I$ of a solution $\varphi_{E}$ to

$$
\left\{\begin{array}{l}
\varphi_{E} \in H_{\mathrm{per}}^{1}\left(M ; \mathbb{R}^{3}\right) \\
b_{Y}\left(\varphi_{E}, \psi\right)=-b_{Y}(E \cdot \psi) \quad \forall \psi \in H_{\mathrm{per}}^{1}\left(M ; \mathbb{R}^{3}\right)
\end{array}\right.
$$

belongs to $C_{Y}$. Lastly, when $J \in\{6,8\}$, as $0=-b_{Y}(E \cdot, \varphi) \forall \varphi \in H_{Y}$, we deduce that $C_{Y}=$ $H_{\mathrm{per}}^{1}\left(Y ; \mathbb{R}^{3}\right)$.

Then we may define $v_{E}^{b}$ by

$$
v_{E}^{b} \in C_{Y} ; \quad a_{Y}\left(v_{E}^{b}, v_{E}^{b}-\psi\right)=-a_{Y}\left(E \cdot, v_{E}^{b}-\psi\right) \quad \forall \psi \in C_{Y}
$$

which does exist and is unique up to an element of $\mathbb{R}^{3}$ by the Stampacchia theorem and also satisfies

$$
a_{Y}\left(v_{E}^{b}, \varphi\right)=-a_{Y}(E \cdot, \varphi) \quad \forall \varphi \in \operatorname{ker} b_{Y}
$$

Thus $v_{E}^{b}-v_{E}^{a}$ belongs to $V_{Y}$ because

$$
\forall \varphi \in \operatorname{ker} b_{Y}, \quad a_{Y}\left(v_{E}^{b}-v_{E}^{a}, \varphi\right)=-a_{Y}(E \cdot, \varphi)+a_{Y}(E \cdot, \varphi)=0 .
$$


Hence we define $B^{\text {eff }}$ in $\operatorname{Lin}\left(\mathbb{S}^{3}\right)$ by

$$
B^{\mathrm{eff}} E \cdot E=b_{Y}\left(E \cdot+v_{E}^{b}, E \cdot+v_{E}^{b}\right) \quad \forall E \in \mathbb{S}^{3} ;
$$

note that $B^{\text {eff }}$ vanishes when $J \in\{3,6,8\}$, that is to say when the viscoelastic phase occupies a nonconnected region.

Then, proceeding as for $A_{s}$, it is straight forward to check that the operator $A_{Y}$ defined by

$$
\begin{aligned}
& D\left(A_{Y}\right)=\left\{\varphi \in V_{Y} ; \exists ! w(\varphi) \in V_{Y} ; b_{Y}(w(\varphi), \psi)+a_{Y}(\varphi, \psi)=0 \forall \psi \in V_{Y}\right\}, \\
& A_{Y}(\varphi)=w(\varphi)
\end{aligned}
$$

is $m$-dissipative, so that the evolution equation

$$
\left\{\begin{array}{l}
\frac{\mathrm{d} z_{E}}{\mathrm{~d} t}=A_{Y} z_{E}, \\
z_{E}(0)=v_{E}^{b}-v_{E}^{a}
\end{array}\right.
$$

has a unique generalized solution in $C^{0}\left([0,+\infty) ; V_{Y}\right) \cap C^{\infty}\left((0,+\infty) ; V_{Y}\right) \cap L^{\infty}\left(0,+\infty ; V_{Y}\right)$ which, for all $p$ in $(0,+\infty)$, does have a Laplace transform satisfying

$$
a_{Y}\left(\mathcal{L} z_{E}, \varphi\right)+b_{Y}\left(p \mathcal{L} z_{E}+v_{E}^{a}-v_{E}^{b}, \varphi\right)=0 \quad \forall \varphi \in H_{Y},
$$

because $\mathcal{L} z_{E}$ belongs to $V_{Y}$. As $v_{E}^{b}$ belongs to $C_{Y}$ and $c_{Y}(p)$ is coercive on $H_{Y} / \mathbb{R}$, (3.15) implies that up to an element of $\mathbb{R}^{3}$

$$
v_{E}^{c(p)}=p z_{E}+v_{E}^{a} .
$$

Let $K(t) \in \operatorname{Lin}\left(\mathbb{S}^{3}\right)$ be defined by

$$
K(t) E \cdot E=a_{Y}\left(z_{E}, E \cdot\right)+b_{Y}\left(\dot{z}_{E}, E \cdot\right) \quad \forall E \in \mathbb{S}^{3},
$$

then (3.25), (3.26) yield

$$
C^{\mathrm{eff}}(p)=\mathcal{L} K(p)+B^{\mathrm{eff}}+\frac{1}{p} A^{\mathrm{eff}} \quad \forall p \in(0,+\infty) .
$$

Last step (Asymptotic behavior of $u_{s}$ ). The fact that $C^{\text {eff }}(p)$ differs from $A^{\text {eff }} / p+B^{\text {eff }}$ implies that the effective behavior of the media is no longer of Kelvin-Voigt type. More precisely, Proposition 3.1 and (3.25) infer that for all $p$ in $(0,+\infty) \mathcal{L} \tilde{u}_{s}(p)$ converges weakly in $H^{1}\left(\Omega ; \mathbb{R}^{3}\right)$ when $J \leqslant 4$, weak-* in $B D(\Omega)$ and strongly in $L^{2}\left(\Omega ; \mathbb{R}^{3}\right)$ when $J \geqslant 5$, toward the unique solution $\breve{u}(p)$ to

$$
\left\{\begin{array}{l}
\check{u}(p) \in H_{\Gamma_{0}}^{1}\left(\Omega ; \mathbb{R}^{3}\right) ; \\
\int_{\Omega} A^{\text {eff }} e(\check{u}(p)) \cdot e(v) \mathrm{d} x+\int_{\Omega}\left[B^{\text {eff }}+\mathcal{L} K(p)\right] e\left(p \check{u}(p)-\bar{u}_{0}\right) \cdot e(v) \mathrm{d} x \\
\quad=\int_{\Omega} \mathcal{L} \tilde{f} \cdot v \mathrm{~d} x+\int_{\Gamma_{1}} \mathcal{L} \tilde{g} \cdot v \mathrm{~d} H_{2} \quad \forall v \in H_{\Gamma_{0}}^{1}\left(\Omega ; \mathbb{R}^{3}\right) .
\end{array}\right.
$$

And as the Laplace transform is one-to-one, (3.7) yields the convergence results. 
Theorem 3.1. If the initial state of the body and the loading satisfy (3.10) and

$$
\begin{cases}(f, g) \in C^{0}\left([0, T] ; L^{2}\left(\Omega ; \mathbb{R}^{3}\right) \times L^{2}\left(\Gamma_{1} ; \mathbb{R}^{3}\right)\right) & \text { when } J \in\{1,4,5,7\}, \\ (f, g) \in C^{1, \alpha}\left([0, T] ; L^{2}\left(\Omega ; \mathbb{R}^{3}\right) \times L^{2}\left(\Gamma_{1} ; \mathbb{R}^{3}\right)\right) & \text { when } J \in\{2,3,6,8\} \text { for } \alpha \text { in }(0,1),\end{cases}
$$

respectively, then, when s goes to $\bar{s}$, the solution $u_{s}$ of $\left(P_{s}\right)$ converges weak-* in $W^{1, \infty}\left(0, T ; H_{\Gamma_{0}}^{1}\left(\Omega ; \mathbb{R}^{3}\right)\right)$ if $J \in\{1,4\}$, in $L^{\infty}\left(0, T ; H_{\Gamma_{0}}^{1}\left(\Omega ; \mathbb{R}^{3}\right)\right)$ if $J \in\{2,3\}$, in $W^{1, \infty}\left(0, T ; B D(\Omega) \cap L^{2}\left(\Omega ; \mathbb{R}^{3}\right)\right)$ if $J \in\{5,7\}$, in $L^{\infty}\left(0, T ; B D(\Omega) \cap L^{2}\left(\Omega ; \mathbb{R}^{3}\right)\right)$ if $J \in\{6,8\}$ toward the unique solution $\bar{u}$ to

$$
\left\{\begin{array}{l}
\bar{u} \in C^{1}\left((0, T] ; H_{\Gamma_{0}}^{1}\left(\Omega ; \mathbb{R}^{3}\right)\right) ; \quad \bar{u}(0)=u^{o}, \\
\int_{\Omega}\left(A^{\text {eff }} e(\bar{u})+B^{\text {eff }} e(\dot{\bar{u}})+\int_{0}^{t} K(t-\tau) e(\dot{\bar{u}})(\tau) \mathrm{d} \tau\right) \cdot e(v) \mathrm{d} x \\
\quad=\int_{\Omega} f \cdot v \mathrm{~d} x+\int_{\Gamma_{1}} g \cdot v \mathrm{~d} H_{2} \quad \forall(v, t) \in H_{\Gamma_{0}}^{1}\left(\Omega ; \mathbb{R}^{3}\right) \times(0, T]
\end{array}\right.
$$

Hence, as in the case $J=1$ studied in [4], the effective behavior of the body is no longer of KelvinVoigt type but rather of viscoelastic type with memory. When $J \in\{1,4,5,7\}$, ker $b_{Y}=\{0\}$ so that $A_{Y}$ is bounded and consequently $K$ decreases exponentially fast with time, hence the effective behavior is viscoelastic with fading memory. When $J \in\{3,6,8\} B^{\text {eff }}$ vanishes.

The case of bilateral contact with purely linear viscous friction $\left(J=6\right.$ with $\left.\mu_{e}^{*}=0\right)$ considered in [5] by the asymptotic expansion method can be rigorously handled by our method through the standard change of unknown $u_{s} \mapsto v_{s}, v_{s}(t)=\exp (-t) u_{s}(t) \forall t \in[0, T]$.

Finally to keep the analysis of reasonable length, we did not consider "pathological cases" like $\left(\bar{\lambda}_{e}, \bar{\mu}_{e}, \bar{\lambda}_{v}, \bar{\mu}_{v}\right) \in\{0,+\infty\}^{4}$ when $J \in\{7,8\}$. Based on the discussion of [6] and [8] about the nature of the mechanical constraint equivalent to a soft viscoelastic layer, the effective behavior will be of viscoelastic type with memory except when $J=8$, and $\bar{\mu}_{v}=\bar{\lambda}_{v}=0$ or $\bar{\mu}_{e}=+\infty$, or $\bar{\mu}_{v}=+\infty$, where it should be elastic.

\section{Proof of Proposition 3.1}

It suffices to only consider the convergence of $u_{s}^{o}$ and case $J=7$. Clearly $u_{s}^{o}$ is the only minimizer of the functional

$$
v \in H_{\Gamma_{0}}^{1}\left(\Omega ; \mathbb{R}^{3}\right) \mapsto F_{s}(v):=\frac{1}{2} a_{s}(v, v)-\int_{\Omega} f^{o} \cdot v \mathrm{~d} x-\int_{\Gamma_{1}} g^{o} \cdot v \mathrm{~d} H_{2} .
$$

One proceeds in four steps:

First step (A compactness property).

Lemma 4.1. Let $\left(v_{s}\right)$ be a sequence in $H_{\Gamma_{0}}^{1}\left(\Omega ; \mathbb{R}^{3}\right)$ such that $F_{s}\left(v_{s}\right) \leqslant C$, then there exists $v$ in $H_{\Gamma_{0}}^{1}\left(\Omega ; \mathbb{R}^{3}\right)$ and a nonrelabeled subsequence such that $v_{s}$ weak $*$ converges in $B D(\Omega)$ and strongly in $L^{2}\left(\Omega ; \mathbb{R}^{3}\right)$ toward some $v$ in $H_{\Gamma_{0}}^{1}\left(\Omega ; \mathbb{R}^{3}\right)$. 
Proof. The very definition of the extension $\tilde{v}_{s}$ of $v_{s},(3.4)$ and (3.6) (see Section 3, First step) imply

$$
\begin{aligned}
\frac{1}{2} a_{s}\left(v_{s}, v_{s}\right) & \leqslant C+\left|f^{o}\right|_{L^{2}\left(\Omega ; \mathbb{R}^{3}\right)}\left|v_{s}\right|_{L^{2}\left(\Omega ; \mathbb{R}^{3}\right)}+\int_{\Gamma_{1}} g^{o} \cdot \tilde{v}_{s} \mathrm{~d} H_{2} \\
& \leqslant C\left(1+\left|f^{o}\right|_{L^{2}\left(\Omega ; \mathbb{R}^{3}\right)}+\left|g^{o}\right|_{L^{2}\left(\Gamma_{1} ; \mathbb{R}^{3}\right)}\right) a_{s}\left(v_{s}, v_{s}\right)^{1 / 2} .
\end{aligned}
$$

Thus $a_{s}\left(v_{s}, v_{s}\right)$ is bounded and the sought after result stems from Remark 3.1.

Second step (More about $\left(P_{E}^{a}\right)$ ). If $v_{E}^{a}$ still denotes the extension into $\mathbb{R}^{3}$ by $Y$-periodicity of the solution $v_{E}^{a}$ to $\left(P_{E}^{a}\right)$, let $\sigma_{E}^{a}$ be the $Y$-periodic field such that

$$
\sigma_{E}^{a}(y)= \begin{cases}A_{M}(y)\left(E+e\left(v_{E}^{a}\right)(y)\right) & \text { a.e. } y \in M \\ A_{I}(y)\left(E+e\left(v_{E}^{a}\right)(y)\right) & \text { a.e. } y \in I\end{cases}
$$

It is easy to check that $\sigma_{E}^{a}$ satisfies $\operatorname{div} \sigma_{E}^{a}=0$ in the sense of distribution in $\mathbb{R}^{3}$ and that $\sigma_{E}^{a}(\cdot / \varepsilon)$ weakly converges in $L^{2}\left(\Omega ; \mathbb{S}^{3}\right)$ toward $\int_{Y} \sigma_{E}^{a}(y) \mathrm{d} y$. For almost $y$ in $Y$, let the strictly convex quadratic form $W_{s^{\prime}}$ be defined on $\mathbb{S}^{3}$ by

$$
W_{s^{\prime}}(y, e):= \begin{cases}\frac{1}{2} A_{M}(y) e \cdot e & \text { if } y \in M_{h} \\ \frac{\lambda_{e}}{2}(\operatorname{tr} e)^{2}+\mu_{e}|e|^{2} & \text { if } y \in B_{h} \\ \frac{1}{2} A_{I}(y) e \cdot e & \text { if } y \in I_{h}\end{cases}
$$

Then the problem

$$
\operatorname{Min}\left\{\int_{Y} W_{s^{\prime}}(y, E+e(\varphi)(y)) \mathrm{d} y ; \varphi \in H_{\text {per }}^{1}\left(Y ; \mathbb{R}^{3}\right)\right\}
$$

has a unique solution $v_{E}^{a, s^{\prime}}$ satisfying $\int_{Y} v_{E}^{a, s^{\prime}}(y) \mathrm{d} y=0$ and whose extension into $\mathbb{R}^{3}$ by $Y$-periodicity is still denoted by $v_{E}^{a, s^{\prime}}$. Let $\sigma_{E}^{a, s^{\prime}}$ defined by

$$
\sigma_{E}^{a, s^{\prime}}(y)=D W_{s^{\prime}}\left(y, E+e\left(v_{E}^{a, s^{\prime}}\right)(y)\right) \quad \text { a.e. } y \in \mathbb{R}^{3},
$$

which clearly satisfies $\operatorname{div} \sigma_{E}^{a, s^{\prime}}=0$ in the sense of distributions in $\mathbb{R}^{3}$. Actually, $\left(P_{E}^{a, s^{\prime}}\right)$ is a kind of approximation of $\left(P_{E}^{a}\right)$ in the sense that, by arguing as in [1,7], one has

$$
\left\{\begin{array}{l}
\lim _{s^{\prime} \rightarrow 0}\left|v_{E}^{a, s^{\prime}}-v_{E}^{a}\right|_{L^{2}\left(Y ; \mathbb{R}^{3}\right)}=0, \\
\lim _{s^{\prime} \rightarrow 0} \int_{Y} W_{s^{\prime}}\left(E+e\left(v_{E}^{a, s^{\prime}}\right)(y)\right) \mathrm{d} y=\frac{1}{2} a_{Y}\left(E \cdot+v_{E}^{a}, E \cdot+v_{E}^{a}\right)=\frac{1}{2} A^{\mathrm{eff}} E \cdot E, \\
\lim _{s^{\prime} \rightarrow 0}\left|\sigma_{E}^{a, s^{\prime}}-\sigma_{E}^{a}\right|_{L^{2}\left(Y ; \mathbb{R}^{3}\right)}=0
\end{array}\right.
$$


and consequently

$$
\lim _{s \rightarrow s^{\prime}} \int_{\Omega}\left|\sigma_{E}^{a, s^{\prime}}(x / \varepsilon)-\sigma_{E}^{a}(x / \varepsilon)\right|^{2} \mathrm{~d} x=0 .
$$

Now we add some ingredients of the mathematical theory of bonded joints $([1,7])$ to the classical proof by [2] for homogenization in elasticity.

Third step (Upper bound for $F_{s}\left(v_{s}\right)$ ).

Lemma 4.2. For all $v$ in $H_{\Gamma_{0}}^{1}\left(\Omega ; \mathbb{R}^{3}\right)$ there exists a sequence $\left(v_{s}\right)$ in $H_{\Gamma_{0}}^{1}\left(\Omega ; \mathbb{R}^{3}\right)$ such that $v_{s}$ weak-* converges in $B D(\Omega)$ and strongly in $L^{2}\left(\Omega ; \mathbb{R}^{3}\right)$ toward $v$ and

$$
\begin{aligned}
F^{\mathrm{eff}}(v) & :=\frac{1}{2} \int_{\Omega} A^{\mathrm{eff}} e(v) \cdot e(v) \mathrm{d} x-\int_{\Omega} f^{o} \cdot v \mathrm{~d} x-\int_{\Gamma_{1}} g^{o} \cdot v \mathrm{~d} H_{2} \\
& =\lim _{s \rightarrow \bar{s}} F_{s}\left(v_{s}\right) .
\end{aligned}
$$

Proof. First we assume that $v$ is affine: $v(x)=E x+d, E \in \mathbb{S}^{3}, d \in \mathbb{R}^{3}$. Let $w_{E, s}$ such that

$$
w_{E, s}(x)=\varepsilon v_{E}^{a, s^{\prime}}(x / \varepsilon) \quad \text { a.e. } x \in \Omega,
$$

then the field $v_{s}:=v+w_{E, s}$ belongs to $H^{1}\left(\Omega ; \mathbb{R}^{3}\right)$, converges weak-* in $B D(\Omega)$ and strongly in $L^{2}\left(\Omega ; \mathbb{R}^{3}\right)$ toward $v$ by due account of (4.4) and the definition of $v_{E}^{a, s^{\prime}}$. Moreover, as

$$
\frac{1}{2} a_{s}\left(v_{s}, v_{s}\right)=|\Omega| \int_{Y} W_{s^{\prime}}\left(E+e\left(v_{E}^{a, s^{\prime}}\right)(y)\right) \mathrm{d} y+\mathrm{o}(\varepsilon)
$$

(4.4) gives $\lim _{s \rightarrow \bar{s}} \frac{1}{2} a_{s}\left(v_{s}, v_{s}\right)=\frac{1}{2} \int_{\Omega} A^{\text {eff }} e(v) \cdot e(v) \mathrm{d} x$. Next we take $v$ as a continuous piecewise-affine function $v(x)=E^{i} x+d^{i}$ on $\Omega^{i}, i$ belonging to a finite set $\mathcal{I}$ of indices, where $\left\{\Omega^{i}\right\}_{i \in \mathcal{I}}$ forms a partition by polyhedral sets. As in the first step, we define $v_{s}$ by $v_{s}^{i}=v+w_{E^{i}, s}$ on each $\Omega^{i}$, but by due account of possible discontinuities on the interface $\sum^{j k}$ between $\Omega^{j}$ and $\Omega^{k}$ we need to introduce $\phi_{\delta}$ in $W^{1, \infty}(\Omega)$, $0 \leqslant \phi_{\delta} \leqslant 1, \phi_{\delta}=1$ on $\sum_{\delta}^{j k}:=\left\{x \in \Omega ; \operatorname{dist}\left(x, \sum_{i}^{j k}\right)<\delta\right\}, \delta>0, \phi_{\delta}=0$ on $\Omega \backslash \sum_{2 \delta}^{j k}$ and $v_{\delta_{s}}=\phi_{\delta} v+\left(1-\phi_{\delta}\right) v_{s}^{i}$ on $\Omega^{i}$. Hence we can repeat the end of the proof by [2], pp. 47-48, because $v_{s}^{i}$ converges strongly toward $v$ in $L^{2}\left(\Omega ; \mathbb{R}^{3}\right)$ and $A_{I}, A_{M}, \lambda_{e}, \mu_{e}$ are bounded.

Eventually the proof of the convergence of $\frac{1}{2} a_{s}\left(v_{s}, v_{s}\right)$ toward $\frac{1}{2} \int_{\Omega} A^{\text {eff }} e(v) \cdot e(v) \mathrm{d} x$ is completed by diagonalization and density arguments.

To tackle loading term $g^{o}$ into account, it suffices to introduce $w_{E, s}$ only on $\bigcup_{j \in J_{\varepsilon}} \varepsilon(j+Y)$, while the convergence of the term involving $f^{o}$ stems from the strong convergence of $v_{s}$ toward $v$ in $L^{2}\left(\Omega ; \mathbb{R}^{3}\right)$.

Fourth step (Lower bound for $F_{s}\left(v_{s}\right)$ ).

Lemma 4.3. For all $v$ in $H_{\Gamma_{0}}^{1}\left(\Omega ; \mathbb{R}^{3}\right)$ and all sequences $\left(v_{s}\right)$ in $H_{\Gamma_{0}}^{1}\left(\Omega ; \mathbb{R}^{3}\right)$ which weak-* converges in $B D(\Omega)$ and strongly in $L^{2}\left(\Omega ; \mathbb{R}^{3}\right)$ toward $v$, we have

$$
F^{\mathrm{eff}}(v) \leqslant \lim _{s \rightarrow \bar{s}} \inf F_{s}\left(v_{s}\right)
$$


Proof. Once more we proceed by introducing a continuous piecewise-affine function $w=E^{i}+d^{i}$ as approximation in $H^{1}$ of $v$ on $\Omega^{i}$. For each $\Omega^{i}$, let $\phi^{i} \in \mathcal{D}\left(\Omega^{i}\right)$ such that $0 \leqslant \phi^{i} \leqslant 1$.

By using functions $w_{E^{i}, s}$ defined like (4.7), the subdifferential inequality yields

$$
\begin{aligned}
\frac{1}{2} a_{s}\left(v_{s}, v_{s}\right) \geqslant \sum_{i \in \mathcal{I}}[ & \int_{\Omega^{i}} \phi^{i}(x) W_{s^{\prime}}\left(x / \varepsilon, E^{i}+e\left(w_{E^{i}, s}\right)(x)\right) \mathrm{d} x \\
& \left.+\int_{\Omega^{i}} \phi^{i}(x) D W_{s^{\prime}}\left(x / \varepsilon, E^{i}+e\left(w_{E^{i}, s}\right)(x)\right) \cdot e\left(v_{s}-w-w_{E^{i}, s}\right) \mathrm{d} x\right] .
\end{aligned}
$$

A slight and obvious modification of the argument used at the beginning of the proof of Lemma 4.2 gives

$$
\lim _{s \rightarrow \bar{s}} \int_{\Omega^{i}} \phi^{i}(x) W_{s^{\prime}}\left(x / \varepsilon, E^{i}+e\left(w_{E^{i}, s}\right)\right)(x) \mathrm{d} x=\frac{1}{2} \int_{\Omega^{i}} \phi^{i}(x) A^{\text {eff }} e(w) \cdot e(w) \mathrm{d} x .
$$

Taking into account (4.3), $\operatorname{div} \sigma_{E^{i}}^{a, s^{\prime}}=0$ and (4.5), one has

$$
\begin{aligned}
\lim _{s \rightarrow \bar{s}} & \int_{\Omega^{i}} \phi^{i}(x) D W_{s^{\prime}}\left(x / \varepsilon, E^{i}+e\left(w_{E^{i}, s}\right)\right) \cdot e\left(v_{s}-w-w_{E^{i}, s}\right) \mathrm{d} x \\
= & \lim _{s \rightarrow \bar{s}} \int_{\Omega^{i}} \phi^{i}(x) \sigma_{E^{i}}^{a, s^{\prime}}(x / \varepsilon) \cdot e\left(v_{s}-w-w_{E^{i}, s}\right) \mathrm{d} x \\
= & \lim _{s \rightarrow \bar{s}}\left(\int_{\Omega^{i}} \sigma_{E^{i}}^{a, s^{\prime}}(x / \varepsilon) \cdot e\left(\phi^{i}\left(v_{s}-w-w_{E^{i}, s}\right)\right) \mathrm{d} x\right. \\
& \left.-\int_{\Omega^{i}} \sigma_{E^{i}}^{a, s^{\prime}}(x / \varepsilon) \cdot \nabla \phi^{i} \otimes_{S}\left(v_{s}-w-w_{E^{i}, s}\right) \mathrm{d} x\right) \\
= & -\lim _{s \rightarrow \bar{s}} \int_{\Omega^{i}} \sigma_{E^{i}}^{a, s^{\prime}}(x / \varepsilon) \cdot \nabla \phi^{i} \otimes_{S}\left(v_{s}-w-w_{E^{i}, s}\right) \mathrm{d} x \\
= & -\lim _{s \rightarrow \bar{s}} \int_{\Omega^{i}} \sigma_{E^{i}}^{a}(x / \varepsilon) \cdot \nabla \phi^{i} \otimes_{S}\left(v_{s}-w-w_{E^{i}, s}\right) \mathrm{d} x \\
= & -\int_{\Omega^{i}}\left(\int_{Y} \sigma_{E^{i}}^{a}(y) \mathrm{d} y\right) \cdot \nabla \phi^{i} \otimes_{S}(v-w) \mathrm{d} x
\end{aligned}
$$

because of the strong convergence in $L^{2}\left(\Omega ; \mathbb{R}^{3}\right)$ of $v_{s}-w-w_{E^{i}, s}$ toward $v-w$. Hence

$$
\lim _{s \rightarrow \bar{s}} \inf \frac{1}{2} a_{s}\left(v_{s}, v_{s}\right) \geqslant \frac{1}{2} \sum_{i \in \mathcal{I}} \int_{\Omega^{i}} \phi^{i} A^{\mathrm{eff}} e(w) \cdot e(w) \mathrm{d} x+\sum_{i \in \mathcal{I}} \int_{\Omega^{i}} \phi^{i} A^{\mathrm{eff}} e(w) \cdot e(v-w) \mathrm{d} x .
$$

As in [2], by letting $\phi^{i}$ converge increasingly to 1 on $\Omega^{i}$ for the first term, and by using (3.17) and the density of piecewise-affine functions in $H^{1}\left(\Omega ; \mathbb{R}^{3}\right)$ for the second term, we conclude that

$$
\lim _{s \rightarrow \bar{s}} \inf \frac{1}{2} a_{s}\left(v_{s}, v_{s}\right) \geqslant \frac{1}{2} \int_{\Omega} A^{\text {eff }} e(v) \cdot e(v) \mathrm{d} x .
$$


Lastly, the arguments used in the proof of Lemma 4.1 (Remark 3.1) yield

$$
\begin{aligned}
\lim _{s \rightarrow \bar{s}} \int_{\Omega} f^{o} \cdot v_{s} \mathrm{~d} x+\int_{\Gamma_{1}} g^{o} \cdot v_{s} \mathrm{~d} H_{2} & =\lim _{s \rightarrow \bar{s}} \int_{\Omega} f^{o} \cdot v_{s} \mathrm{~d} x+\int_{\Gamma_{1}} g^{o} \cdot \tilde{v}_{s} \mathrm{~d} H_{2} \\
& =\int_{\Omega} f^{o} \cdot v \mathrm{~d} x+\int_{\Gamma_{1}} g^{o} \cdot v \mathrm{~d} H_{2} .
\end{aligned}
$$

Thus Proposition 3.1 stems classically (see [2,3]) from a combination of Lemmas 4.1-4.3.

\section{References}

[1] A. Ait-Moussa, A modelling of elastic bonded joints, Modélisation et étude des singularités de contraintes d'un joint collé très mince, $\mathrm{PhD}$ thesis, Université Montpellier II, France, 1989.

[2] H. Attouch, Variational Convergence for Functions and Operators, Applicable Mathematics Series, Pitman Advanced Publishing Program, 1984.

[3] H. Attouch, G. Buttazzo and G. Michaille, Variational Analysis in Sobolev and BV Spaces: Application to PDEs and Optimization, MPS-SIAM Book Series on Optimization, 2005.

[4] G. Francfort and P. Suquet, Homogenization and mechanical dissipation in thermoviscoelasticity, Arch. Rational Mech. Anal. 96(3) (1986), 265-293.

[5] F. Lénée, Contribution à l' étude des matériaux composites et de leur endommagement, Thèse doctorat d' état, Université Paris VI, 1984.

[6] C. Licht, A. Léger, S. Orankitjaroen and A.O. Khaoua, Dynamics of elastic bodies connected by a thin soft viscoelastic layer, J. Math. Pures Appl. 99(6) (2013), 685-703.

[7] C. Licht and G. Michaille, A modelling of elastic bonded joints, Advances in Mathematical Sciences and Applications 7 (1997), 711-740.

[8] C. Licht and S. Orankitjaroen, Dynamics of elastic bodies connected by a thin soft inelastic layer, C. R. Mecanique 341(3) (2013), 323-332.

[9] P.K. Mallick, Fiber-Reinforced Composites: Materials, Manufacturing, and Design, 3rd edn, CRC Press, Taylor \& Francis Group, 2007.

[10] E. Sanchez-Palencia, Non-homogeneous Media and Vibration Theory, Lecture Notes in Physics, Vol. 127, Springer, 2009.

[11] L. Tartar, The General Theory of Homogenization, A Personalized Introduction, Lecture Notes of the Unione Mathematica Italiana, Vol. 7, Springer, 2009.

[12] R. Temam, Mathematical Problem in Plasticity, Gauthier-Villars, 1985. 